\title{
Frecuencias alélicas, genotípicas y haplotípicas HLA-A, HLA-B, HLA-DRB1 en donantes fallecidos, Medellín, Colombia
}

\author{
Libia M. Rodríguez, Mabel C. Giraldo, Natalia García, Laura Velásquez, Sara C. París, \\ Cristiam M. Álvarez, Luis F. García \\ Grupo de Inmunología Celular e Inmunogenética, Facultad de Medicina, Universidad de Antioquia, \\ Medellín, Colombia
}

Introducción. La caracterización genética del sistema HLA es de gran utilidad en estudios antropogenéticos, en la comprensión de mecanismos asociados a susceptibilidad o resistencia a diversas enfermedades, en los fenómenos inmunológicos durante el embarazo y en la selección de donantes/receptores en trasplantes de órganos.

Objetivo. Determinar las frecuencias alélicas, genotípicas y haplotípicas HLA-A, -B, -DRB1 en donantes fallecidos en Medellín.

Materiales y métodos. Se incluyeron 926 donantes entre febrero de 1989 y septiembre de 2006, a los cuales se les realizó la tipificación HLA-A, -B, -DRB1 por PCR-SSP (single specific primer-polymerase chain reaction) de mediana resolución. Las frecuencias alélicas, genotípicas y haplotípicas fueron estimadas mediante el algoritmo de máxima verosimilitud. Se evaluó el ajuste al equilibrio de Hardy-Weimberg por una prueba exacta análoga a la de Fisher usando la cadena de Markov, así como el desequilibrio de ligamiento entre pares de loci.

Resultados. Se identificaron 22, 43 y 14 alelos para los loci HLA-A, -B, -DRB, respectivamente, de los cuales los más frecuentes fueron: $A^{\star} 02, A^{\star} 24, B^{*} 35$ y DRB1*04. En los loci HLA-A y -B se observó deficiencia en la frecuencia de heterocigóticos esperada $(p<0,01$ y $p<0,00001$, respectivamente). Los haplotipos más frecuentes fueron HLA-A*24, $B^{\star} 35$ (7,7\%), HLA-B*35 DRB1*04 (6,4\%) y HLA-A*24, DRB1*04 (8,9\%) para HLA-A, -DRB1, y para 3 loci fueron HLA$A^{\star} 24, B^{\star} 35, \mathrm{DRB} 1{ }^{*} 04(4,6 \%)$ y HLA-A*24, B*61, DRB1*04 (2,0\%).

Conclusiones. Estos resultados corroboran la composición triétnica de nuestra población, en la cual predomina el grado de mezcla caucásica, a diferencia de otras latinoamericanas, y podrán ser usados como referencia para otros estudios y aplicaciones en esta población.

Palabras clave: antígenos HLA/genética/análisis, frecuencia de los genes, haplotipos, genotipo, prueba de histocompatibilidad, humanos.

\section{Human leucocyte antigen gene (HLA-A, HLA-B, HLA-DRB1) frequencies in deceased organ} donors

Introduction. Genetic characterization of the human leucocyte antigen (HLA) system has provided insights into mechanisms of susceptibility to diverse diseases and immunological phenomena during pregnancy, as well as providing evidence for compatibility in the selection of organ transplant donors and recipients.

Objective. The HLA-A,-B,-DRB1 allele, genotype and haplotype frequencies were determined in deceased organ donors in Medellín, Colombia.

Materials and methods. The genotypes of 926 deceased donors were evaluated over a 17year period (1989-2006). HLA-A, HLA-B and HLA-DRB1 typing was performed by sequence specific primer-polymerase chain reaction (SSP-PCR). Maximum likelihood frequencies were estimated by the zipper version of expectation maximation algorithm. Hardy-Weinberg equilibrium were determined by an exact test analogous to Fisher's test by using Markov's chain, and linkage disequilibrium between pairs of loci. 
Results. Twenty-two, 43 and 14 alleles were identified for HLA-A, -B and -DRB loci, respectively. The most frequent were $A^{*} 02, A^{*} 24, B^{*} 35$, and DRB $1 * 04$. A deficiency in the proportion of heterozygotes in HLA-A and $B$ loci $(p<0.01$ and $p<0.00001$, respectively). The most frequent haplotypes were as follows: HLA-A*24, $B^{\star} 35(7.7 \%)$ for HLA-A,-B; HLA-B*35, DRB1*04 (6.4\%) for HLA-B,-DRB1 and HLA-A*24, DRB1 ${ }^{*} 04(8.9 \%)$ for HLA-A,-DRB1. For the 3 loci HLA-A,-B,DRB1, the most frequent haplotypes were $A^{*} 24, B^{*} 35$, DRB1*04 (4.6\%) and $A^{*} 24, B^{*} 61$, DRB1*04 (2.0\%).

Conclusions. These results confirm the three-ethnic ancestry of the Medellin population. The predominance of Caucasian admixture differs from many other Latin-American populations and can serve as a reference for comparative studies of these populations as well as applications within the Medellin population.

Key words: HLA antigens/genetics/analysis, gene frequency, haplotypes, genotype, histocompatibility testing, humans.

El sistema de antígenos leucocitarios humanos (HLA, acrónimo en inglés de human leukocyte antigen), codificado por el complejo mayor de histocompatibilidad (CMH), comprende una familia de genes ubicados en el brazo corto del cromosoma 6 y constituye uno de los sistemas más polimorfos del genoma humano (1-3). Su alta variabilidad genética ha sido de gran interés en muchos campos debido a su papel determinante en la respuesta inmune, susceptibilidad o resistencia a un gran número de enfermedades complejas y en la supervivencia de los trasplantes de tejidos (4-15).

Con el desarrollo de diversas técnicas moleculares se ha incrementado el conocimiento de estas moléculas lo que ha permitido la identificación de numerosas variantes del sistema HLA (1,16-22). Por otra parte, las frecuencias de alelos HLA y sus patrones de desequilibrio de ligamiento varían entre diferentes poblaciones humanas por lo cual el estudio del polimorfismo HLA ha sido relevante en estudios antropogenéticos, en la diferenciación interpoblaciones e intrapoblaciones, permitiendo definir grupos étnicos específicos, mezclas raciales, distancias genéticas y patrones de migración (23-35). De igual manera, la caracterización de la constitución genética de estas

\section{Correspondencia}

Luis Fernando García, Grupo de Inmunología Celular e Inmunogenética, Sede de Investigación Universitaria, Universidad de Antioquia, Carrera 53 № 61-30, laboratorio 410.

Teléfono: (+574) 210 6446; fax: (+574) 2106455.

Ifgarcia@udea.edu.co

Recibido: 09/04/07; aceptado: 23/08/07 moléculas en una determinada población es una poderosa herramienta en la práctica clínica para la realización de estudios de asociación HLA con diversas enfermedades $y$, en el caso de los trasplantes de órganos, particularmente trasplantes renales, permite calcular la probabilidad de encontrar un donante en una población particular, compatible para determinado receptor y estimar los tiempos de espera (36-39).

En Colombia se carece de estudios recientes en población general, ya que los publicados hasta el momento muestran frecuencias alélicas basadas en técnicas menos avanzadas, como la serología, o han sido realizados en grupos étnicos específicos (40-44). Dada la importancia genética e inmunológica del sistema HLA, se decidió realizar el presente estudio con el objetivo de determinar las frecuencias alélicas, genotípicas y haplotípicas en donantes fallecidos de Medellín, Colombia. Conocer la estructura genética de la población para los loci HLA es de gran utilidad para los programas de trasplantes de órganos, y en muchas especialidades médicas relacionadas con enfermedades genéticamente asociadas al HLA.

\section{Materiales y métodos \\ Población de estudio}

El presente es un estudio descriptivo en el cual se incluyeron 926 donantes fallecidos de Medellín entre febrero de 1989 y septiembre del 2006, utilizados para diferentes tipos de trasplantes, a los cuales se les realizó la tipificación HLA-A,-B,DRB1 en el Laboratorio de Inmunología de Trasplantes del Grupo de Inmunología Celular e 
Inmunogenética (GICIG) de la Universidad de Antioquia, previo consentimiento informado de los familiares del donante de acuerdo con la legislación vigente sobre donación de órganos y tejidos en Colombia (Decretos 1546 de 1998 y 2493 de 2004).

\section{Clasificación HLA ABDRB mediante PCR-SSP}

La extracción de las muestras de ADN se realizó a partir de las capas de glóbulos blancos obtenidas de bazo o sangre anticoagulada con EDTA, utilizando la técnica de precipitación salina (salting out). La concentración de ADN de cada una de las muestras se ajustó a $150 \mathrm{ng} / \mu \mathrm{l}$ y, luego, se determinaron los genes HLA clase I $(A, B)$ y HLA clase II (DRB) usando el HLA-A+B+DRB Typing Tray Kit de mediana resolución, producido por el Collaborative Transplant Study (CTS, University of Heidelberg, Heidelberg, Alemania). Las reacciones de amplificación se detectaron mediante electroforesis en geles de agarosa al $2 \%$ teñidos con $4 \mu \mathrm{l}$ de bromuro de etidio $(10 \mathrm{mg} /$ $\mathrm{ml}$ ). La asignación alélica se realizó mediante el análisis de los patrones de amplificados empleando las tablas de clasificación suministradas por el fabricante.

\section{Análisis estadístico}

Para el análisis estadístico se realizó previamente en la base de datos, la homologación del grado de resolución de la tipificación HLA a 2 dígitos (45). Las frecuencias alélicas y genotípicas fueron determinadas por estimación de máxima verosimilitud; el desequilibrio de ligamiento (DL) entre pares de loci y el índice de fijación de Fisher se calcularon empleando el paquete de análisis genético Genepop (http://genepop.curtin.edu.au) (46). El equilibrio Hardy-Weinberg para cada locus se probó con el paquete Arlequín, versión 3.0, el cual emplea una prueba exacta análoga a la prueba Fisher, teniendo en cuenta modificaciones al algoritmo de la cadena de Markov, descrito por Guo y Thompson en 1992 (47). Las frecuencias haplotípicas fueron estimadas igualmente en Arlequín, versión 3.0, empleando el algoritmo de máxima verosimilitud versión súper (zipper version of expectation maximation). Para los haplotipos más frecuentes HLA-A, -B, HLA-B, -DRB1 o HLA$A,-D R B$ se calcularon los valores de desequilibrio de ligamiento para cada par de alelos específicamente, según la formula de desequilibrio para alelos múltiples y los valores normalizados de desequilibrio de ligamiento ( $\left.D^{\prime}\right)$, según Lewontin (48); además, se calcularon los coeficientes $r^{2}$. El rango de valores del desequilibrio de ligamiento va de +1 a -1 ; un desequilibrio de ligamiento de 0 indica equilibrio de ligamiento, mientras que un desequilibrio de ligamiento de +1 indica una asociación de un par de alelos presentes en un haplotipo y un valor de -1 indica ausencia de un haplotipo que comprenda esos 2 alelos.

La significancia estadística de los desequilibrios de ligamiento se comprobó mediante la prueba $\chi^{2}$ para dos loci (48).

\section{Resultados}

\section{Características demográficas}

La mayoría de los donantes estudiados eran hombres $(78,9 \%)$, con una edad promedio de $29,6 \pm 12,6$ años (rango, 3 a 66 años). Las causas de muerte fueron: trauma en $752(81,6 \%)$, accidente cerebrovascular en $108(11,7 \%)$, anoxia en $47(5,1 \%)$, tumor cerebral en $6(0,7 \%)$, otras en $9(1,0 \%)$, entre las cuales se encontraban epilepsia, envenenamiento, embolia grasa y edema cerebral. Sólo en cuatro donantes no se encontró información sobre la causa de muerte.

Cuadro 1. Equilibrio Hardy-Weinberg*.

\begin{tabular}{lcccc}
\hline Locus & $\begin{array}{c}\text { Heterocigóticos } \\
\text { observados }\end{array}$ & $\begin{array}{c}\text { Heterocigóticos } \\
\text { esperados }\end{array}$ & $\mathbf{p}$ & Fis $^{\star *}$ \\
\hline HLA-A & 0,828 & 0,884 & 0,00483 & $+0,0632$ \\
HLA-B & 0,842 & 0,934 & 0,00000 & $+0,0982$ \\
HLA-DRB & 0,877 & 0,884 & 0,82911 & $+0,0085$ \\
\hline
\end{tabular}

*Analizado en Genepop, versión web (http://genepop.curtin.edu.au)

${ }^{* *}$ Fis: índice de fijación de Fisher. 


\section{Frecuencias alélicas}

Se observaron 22, 43 y 14 alelos en los loci HLAA, -B, -DRB1, respectivamente. Las frecuencias genotípicas observadas en los loci HLA-A,-B difirieron significativamente de las frecuencias genotípicas esperadas $(p<0,01$ y $p<0,00001$, respectivamente), observándose una deficiencia de individuos heterocigóticos. En contraste, las frecuencias genotípicas en el locus HLA-DRB se encontraron en equilibrio Hardy-Weinberg (cuadro 1). Para los loci HLA-A y B se alcanzaron índices de fijación de Fisher de +0,0632 y 0,0982, respectivamente.

Los alelos más frecuentes en el locus HLA-A fueron: $A^{*} 02(22,2 \%), A^{\star} 24(19,1 \%), A^{*} 03(7,9 \%)$ y $A^{\star} 23(7,2 \%)$, seguidos por $A^{*} 30, A^{*} 68, A^{*} 01$ con una frecuencia mayor al $5 \%$ (cuadro 2 ). Los alelos más frecuentes en el locus HLA-B fueron: $B^{*} 35(17,8 \%), B^{*} 44(9,1 \%)$, seguidos por $B^{*} 18$, $B^{*} 51, B^{*} 61, B^{*} 65, B^{*} 39, B^{*} 07, B^{*} 60$, y $B^{\star} 08$, con frecuencias mayores o iguales a $3 \%$ (cuadro 3 ).

Cuadro 2. Distribución de frecuencias alélicas HLA-A en donantes fallecidos en Medellín.

\begin{tabular}{|c|c|c|}
\hline \multirow[b]{2}{*}{ Alelos HLA-A } & \multicolumn{2}{|c|}{ Frecuencia alélica* } \\
\hline & $\mathbf{N}$ & $\%$ \\
\hline 01 & 94 & 5,1 \\
\hline 02 & 411 & 22,2 \\
\hline 03 & 146 & 7,9 \\
\hline 10 & 2 & 0,1 \\
\hline 11 & 92 & 5,0 \\
\hline 23 & 133 & 7,2 \\
\hline 24 & 354 & 19,1 \\
\hline 25 & 20 & 1,1 \\
\hline 26 & 61 & 3,3 \\
\hline 29 & 69 & 3,7 \\
\hline 30 & 124 & 6,7 \\
\hline 31 & 77 & 4,2 \\
\hline 32 & 33 & 1,8 \\
\hline 33 & 76 & 4,1 \\
\hline 34 & 11 & 0,6 \\
\hline 36 & 8 & 0,4 \\
\hline 43 & 2 & 0,1 \\
\hline 66 & 11 & 0,6 \\
\hline 68 & 103 & 5,6 \\
\hline 69 & 7 & 0,4 \\
\hline 74 & 17 & 0,9 \\
\hline 80 & 1 & 0,1 \\
\hline
\end{tabular}

*La determinación de los alelos HLA-A se realizó por PCRSSP y las frecuencias se estimaron por máxima verosimilitud utilizando el programa Genepop.
En cuanto al locus HLA-DRB1, los alelos más frecuentes fueron: DRB $1{ }^{*} 04(22,1 \%)$, DRB $1 * 13$ $(13,4 \%)$, DRB1*07 $(11,2 \%)$, DRB1*15 $(10,0 \%)$, DRB1*01 (9,1\%), DRB1*17 (7,6\%), DRB1*11 $(7,4 \%)$ (cuadro 4$)$.

Cuadro 3. Distribución frecuencias alélicas HLA-B en donantes fallecidos en Medellín.

\begin{tabular}{|c|c|c|}
\hline \multirow[b]{2}{*}{ Alelos HLA-B } & \multicolumn{2}{|c|}{ Frecuencia alélica } \\
\hline & $\mathbf{N}$ & $\%$ \\
\hline 07 & 89 & 4,8 \\
\hline 08 & 56 & 3,0 \\
\hline 13 & 18 & 1,0 \\
\hline 14 & 6 & 0,3 \\
\hline 15 & 11 & 0,6 \\
\hline 17 & 21 & 1,1 \\
\hline 18 & 131 & 7,1 \\
\hline 27 & 25 & 1,4 \\
\hline 35 & 329 & 17,8 \\
\hline 37 & 5 & 0,3 \\
\hline 38 & 45 & 2,4 \\
\hline 39 & 91 & 4,9 \\
\hline 40 & 21 & 1,1 \\
\hline 41 & 26 & 1,4 \\
\hline 42 & 15 & 0,8 \\
\hline 44 & 168 & 9,1 \\
\hline 45 & 20 & 1,1 \\
\hline 46 & 2 & 0,1 \\
\hline 47 & 4 & 0,2 \\
\hline 48 & 12 & 0,6 \\
\hline 49 & 53 & 2,9 \\
\hline 50 & 28 & 1,5 \\
\hline 51 & 118 & 6,4 \\
\hline 52 & 21 & 1,1 \\
\hline 53 & 39 & 2,1 \\
\hline 55 & 16 & 0,9 \\
\hline 56 & 5 & 0,3 \\
\hline 57 & 46 & 2,5 \\
\hline 58 & 37 & 2,0 \\
\hline 59 & 4 & 0,2 \\
\hline 60 & 62 & 3,4 \\
\hline 61 & 100 & 5,4 \\
\hline 62 & 31 & 1,7 \\
\hline 63 & 31 & 1,7 \\
\hline 64 & 21 & 1,1 \\
\hline 65 & 96 & 5,2 \\
\hline 67 & 2 & 0,1 \\
\hline 70 & 8 & 0,4 \\
\hline 71 & 8 & 0,4 \\
\hline 72 & 26 & 1,4 \\
\hline 75 & 1 & 0,1 \\
\hline 78 & 3 & 0,2 \\
\hline 81 & 1 & 0,1 \\
\hline
\end{tabular}

*La determinación de los alelos HLA-B se realizó por PCRSSP y las frecuencias se estimaron por máxima verosimilitud utilizando el programa Genepop. 
Cuadro 4. Frecuencias alélicas HLA-DRB1 en donantes fallecidos en Medellín.

\begin{tabular}{crr}
\hline & \multicolumn{2}{c}{ Frecuencia alélica } \\
\cline { 2 - 3 } Alelos HLA-DRB1 & \multicolumn{1}{c}{$\mathbf{N}$} & \multicolumn{1}{c}{$\%$} \\
\hline 01 & 169 & 9,1 \\
04 & 410 & 22,1 \\
07 & 207 & 11,2 \\
08 & 104 & 5,6 \\
09 & 25 & 1,4 \\
10 & 38 & 2,1 \\
11 & 137 & 7,4 \\
12 & 19 & 1,0 \\
13 & 248 & 13,4 \\
14 & 80 & 4,3 \\
15 & 185 & 10,0 \\
16 & 64 & 3,5 \\
17 & 141 & 7,6 \\
18 & 25 & 1,4 \\
\hline
\end{tabular}

*La determinación de los alelos HLA-DRB1 se realizó por PCR-SSP y las frecuencias se estimaron por máxima verosimilitud utilizando el programa Genepop.

\section{Frecuencias genotípicas}

En la figura $1 \mathrm{~A}$ se muestran los genotipos para el locus HLA-A con frecuencias mayores al 1,0\%, lo cuales representan el $65 \%$ de los genotipos presentes para este locus. Los genotipos más frecuentes fueron: $A^{*} 02,24(8,2 \%), A^{*} 02,02(5,3 \%)$, $A^{*} 24,24(4,9 \%)$, y $A^{*} 02,03(3,8 \%)$. En la figura $1 B$ se muestran los genotipos para el locus HLA-B con frecuencias mayores al $1 \%$ que representan $27 \%$ del total de genotipos observados. Los genotipos con mayor frecuencia fueron: $B^{\star} 35,35$
$(4,8 \%), B^{\star} 35,44(3,8 \%), B^{\star} 35,61(2,4 \%)$, y $B^{\star} 35,51$ $(2,3 \%)$. De igual manera en la figura $1 \mathrm{C}$ se muestras los genotipos para el locus HLA-DRB1 con frecuencias mayores de $1,0 \%$ (74\% del total de genotipos). Los genotipos más frecuentes fueron: DRB1 ${ }^{*} 04,13(5,9 \%), \mathrm{DRB} 1{ }^{*} 04,07(5,2 \%)$, DRB1*04,15 (5,2\%), DRB1*04,04 (5,0\%) y DRB $1{ }^{*} 01,04(4,2 \%)$.

Cada uno de los pares de loci se encontró en desequilibrio de ligamiento, siendo más significativo el desequilibrio entre los loci HLA-A y HLA-B $(p=0,0099)$, en comparación con el desequilibrio entre los loci HLA-B y HLA-DRB1 $(p=0,0174)$ y entre HLA-A y HLA-DRB $(p=0,0268)$.

\section{Estimación de los haplotipos HLA-A, -B, -DRB1}

Para el análisis de los desequilibrios de ligamientos solo se consideraron los haplotipos con frecuencias iguales o superiores al $1 \%$, los cuales representaron el $40,8 \%, 42,5 \%$ y $48,2 \%$ del total de haplotipos para HLA-A,-B, HLA-B,-DRB y HLA$A$,-DRB1, respectivamente (cuadro 5).

Los haplotipos más comunes fueron: $A * 24 B^{\star} 35$ $(7,7 \%), A^{*} 02 B^{*} 51(3,4 \%)$ para HLA-A,- B; $B^{*} 35$ DRB $1{ }^{*} 04(6,4 \%), B^{*} 44$ DRB1*07 $(3,7 \%), B^{*} 18$ DRB1*17 $(3,6 \%)$ para HLA-B, -DRB1; $A^{*} 24$ DRB1*04 (8,9\%), $A^{*} 02$ DRB1*04 (6,6\%), $A^{*} 02$ DRB1*13 (3,5\%) para HLA-A, -DRB1 (cuadro 5).

Los valores de desequilibrio de ligamiento fueron significativos $(p<0,0001)$ en 13 de los 19 haplotipos HLA-A,-B, en 10 de los 20 haplotipos HLA-B,-
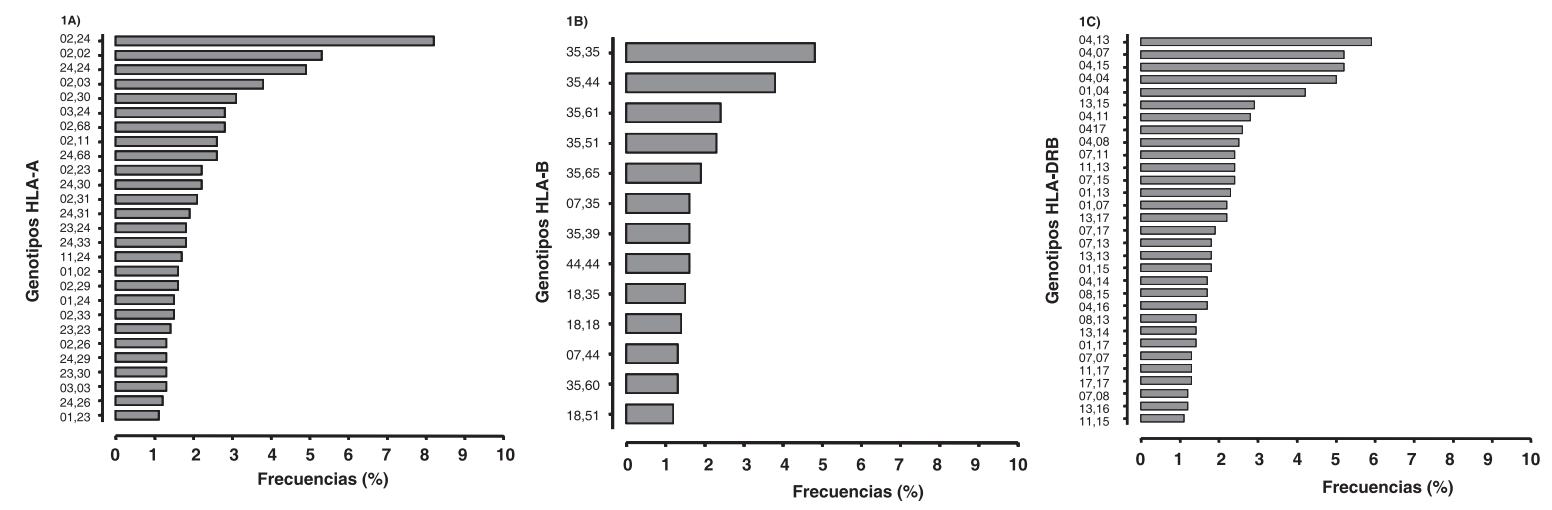

Figura 1. Frecuencias genotípicas: A) HLA-A, B) HLA-B, C) HLA-DRB1, mayores de $1 \%$ en donantes fallecidos en Medellín, $\mathrm{n}=926$. Las frecuencias se estimaron por máxima verosimilitud utilizando el programa Genepop. 
Cuadro 5. Haplotipos más frecuentes en donantes fallecidos en Medellín*.

\begin{tabular}{|c|c|c|c|c|c|}
\hline HLA-A, B & Frecuencias & D & $\mathbf{D}^{\prime}$ & $\mathbf{r}^{2}$ & $\chi^{2}$ \\
\hline A24,B35 & 0,0773 & 0,0434 & 0,3021 & 0,0833 & 154,22 \\
\hline $\mathrm{A} 02, \mathrm{~B} 51$ & 0,0342 & 0,0201 & 0,4048 & 0,0391 & 72,41 \\
\hline A02,B39 & 0,0278 & 0,0169 & 0,4425 & 0,0354 & 65,65 \\
\hline A02,B35 & 0,0241 & $-0,0153$ & $-0,3885$ & 0,0093 & 17,21 \\
\hline A24,B61 & 0,0235 & 0,0132 & 0,3017 & 0,0220 & 40,74 \\
\hline $\mathrm{A} 02, \mathrm{~B} 44$ & 0,0226 & 0,0025 & 0,0351 & 0,0004 & $0,80^{d}$ \\
\hline A03,B35 & 0,0215 & 0,0075 & 0,1158 & 0,0053 & $9,84^{b}$ \\
\hline A33,B65 & 0,0195 & 0,0174 & 0,4470 & 0,1563 & 289,55 \\
\hline$A 30, B 18$ & 0,0195 & 0,0148 & 0,2371 & 0,0531 & 98,28 \\
\hline $\mathrm{A} 02, \mathrm{~B} 18$ & 0,0191 & 0,0034 & 0,0620 & 0,0010 & $1,90^{d}$ \\
\hline A29,B44 & 0,0179 & 0,0145 & 0,4280 & 0,0712 & 131,79 \\
\hline $\mathrm{A} 03, \mathrm{~B} 07$ & 0,0176 & 0,0138 & 0,3117 & 0,0574 & 106,27 \\
\hline A23,B44 & 0,0158 & 0,0093 & 0,1423 & 0,0157 & 29,07 \\
\hline $\mathrm{A} 02, \mathrm{~B} 61$ & 0,0126 & 0,0006 & 0,0147 & 0,0000 & $0,08^{d}$ \\
\hline A24,B07 & 0,0113 & 0,0021 & 0,0542 & 0,0006 & $1,16^{d}$ \\
\hline A26,B38 & 0,0112 & 0,0104 & 0,4426 & 0,1434 & 265,56 \\
\hline $\mathrm{A} 01, \mathrm{~B} 08$ & 0,0111 & 0,0096 & 0,3337 & 0,0648 & 120,00 \\
\hline A23,B49 & 0,0111 & 0,0090 & 0,3408 & 0,0442 & 81,86 \\
\hline A11,B35 & 0,0103 & 0,0015 & 0,0360 & 0,0003 & $0,58^{d}$ \\
\hline HLA-B, DRB & Frecuencias & D & $\mathbf{D}^{\prime}$ & $\mathbf{r}^{2}$ & $\chi^{2}$ \\
\hline B35,DRB04 & 0,0636 & 0,0242 & 0,1752 & 0,0233 & 43,19 \\
\hline B44,DRB07 & 0,0374 & 0,0273 & 0,3387 & 0,0909 & 168,31 \\
\hline B18,DRB17 & 0,0355 & 0,0301 & 0,4611 & 0,1963 & 363,62 \\
\hline B07,DRB15 & 0,0278 & 0,0230 & 0,5314 & 0,1285 & 238,06 \\
\hline B61,DRB04 & 0,0259 & 0,0139 & 0,3308 & 0,0220 & 40,67 \\
\hline B51, DRB04 & 0,0211 & 0,0069 & 0,1401 & 0,0047 & $8,70^{b}$ \\
\hline B35,DRB01 & 0,0200 & 0,0038 & 0,0503 & 0,0012 & $2,18^{d}$ \\
\hline B35, DRB11 & 0,0199 & 0,0067 & 0,1109 & 0,0046 & $8,43^{b}$ \\
\hline B39,DRB04 & 0,0183 & 0,0074 & 0,1931 & 0,0068 & $12,54^{\mathrm{a}}$ \\
\hline B65,DRB01 & 0,0174 & 0,0127 & 0,2690 & 0,0394 & 72,89 \\
\hline B60,DRB04 & 0,0168 & 0,0094 & 0,3603 & 0,0158 & 29,31 \\
\hline B44,DRB15 & 0,0154 & 0,0064 & 0,0779 & 0,0055 & $10,10^{b}$ \\
\hline B35,DRB08 & 0,0153 & 0,0053 & 0,1141 & 0,0036 & $6,65^{c}$ \\
\hline B35,DRB13 & 0,0146 & $-0,0092$ & $-0,3873$ & 0,0050 & $9,27^{b}$ \\
\hline B65,DRB13 & 0,0145 & 0,0075 & 0,1677 & 0,0099 & 18,40 \\
\hline B08,DRB17 & 0,0143 & 0,0120 & 0,4319 & 0,0705 & 130,59 \\
\hline B53,DRB13 & 0,0123 & 0,0095 & 0,5207 & 0,0378 & 70,01 \\
\hline B35,DRB16 & 0,0117 & 0,0055 & 0,1944 & 0,0063 & $11,62^{b}$ \\
\hline B51,DRB13 & 0,0117 & 0,0031 & 0,0567 & 0,0014 & $2,62^{d}$ \\
\hline B35,DRB07 & 0,0111 & $-0,0087$ & $-0,4406$ & 0,0053 & $9,77^{\mathrm{b}}$ \\
\hline HLA-A, DRB & Frecuencias & D & $D^{\prime}$ & r2 & $\chi^{2}$ \\
\hline A24,DRB04 & 0,0889 & 0,0466 & 0,3129 & 0,0814 & 150,67 \\
\hline A02,DRB04 & 0,0661 & 0,0170 & 0,0985 & 0,0097 & 17,91 \\
\hline A02,DRB13 & 0,0347 & 0,0050 & 0,0480 & 0,0013 & $2,32^{d}$ \\
\hline A02,DRB07 & 0,0249 & 0,0001 & 0,0007 & 0,0000 & $0,00^{d}$ \\
\hline A03,DRB04 & 0,0217 & 0,0043 & 0,0701 & 0,0015 & $2,74^{d}$ \\
\hline A03,DRB01 & 0,0207 & 0,0135 & 0,1890 & 0,0304 & 56,30 \\
\hline A24,DRB15 & 0,0202 & 0,0012 & 0,0142 & 0,0001 & $0,18^{d}$ \\
\hline A02,DRB17 & 0,0194 & 0,0025 & 0,0418 & 0,0005 & $0,93^{d}$ \\
\hline A30,DRB17 & 0,0186 & 0,0135 & 0,2179 & 0,0414 & 76,63 \\
\hline A29,DRB07 & 0,0178 & 0,0136 & 0,4115 & 0,0521 & 96,53 \\
\hline A23,DRB07 & 0,0172 & 0,0092 & 0,1439 & 0,0127 & 23,56 \\
\hline A24,DRB16 & 0,0171 & 0,0105 & 0,3756 & 0,0214 & 39,63 \\
\hline A33,DRB13 & 0,0171 & 0,0116 & 0,3257 & 0,0293 & 54,32 \\
\hline A02,DRB08 & 0,0160 & 0,0036 & 0,0812 & 0,0014 & $2,55^{d}$ \\
\hline A03,DRB15 & 0,0155 & 0,0076 & 0,1078 & 0,0090 & 16,58 \\
\hline A02,DRB11 & 0,0147 & $-0,0017$ & $-10,48$ & 0,0003 & $0,46^{d}$ \\
\hline A30,DRB13 & 0,0142 & 0,0052 & 0,0898 & 0,0037 & $6,93^{c}$ \\
\hline A02,DRB14 & 0,0132 & 0,0036 & 0,1073 & 0,0018 & $3,38^{d}$ \\
\hline A02,DRB01 & 0,0123 & $-0,0080$ & $-0,1119$ & 0,0044 & $8,17^{b}$ \\
\hline A24,DRB08 & 0,0121 & 0,0013 & 0,0293 & 0,0002 & $0,40^{d}$ \\
\hline
\end{tabular}

${ }^{a} p<0,001,{ }^{b} p<0,01,{ }^{c} p<0,05,{ }^{d} N S$. Todos los otros desequilibrios $p<0,0001$

* Las frecuencias haplotípicas fueron estimadas mediante el algoritmo de máxima verosimilitud versión super (zipper version of expectation maximation) empleando Arlequín, versión 3.0 


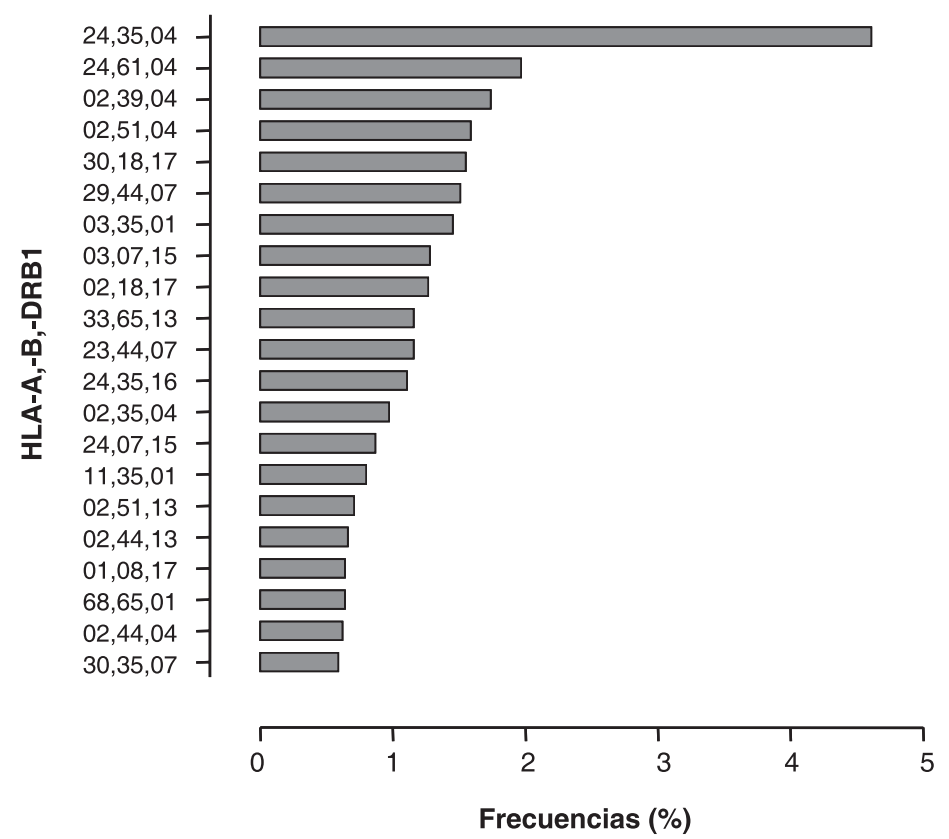

Figura 2. Haplotipos HLA-A,-B,-DRB1 más frecuentes en donantes fallecidos en Medellín, $n=926$. Las frecuencias haplotípicas fueron estimadas mediante el algoritmo de máxima verosimilitud versión super (zipper version of expectation maximation) empleando Arlequín, versión 3.0

DRB1, y en 9 de los 20 haplotipos HLA-A,-DRB1. De estos los valores de $\mathrm{D} \phi$ fueron iguales 0 mayores al $40 \%$ en 5,4 y 9 de los haplotipos HLA-A,-B, HLA-B,-DRB1, HLA-A,-DRB1 respectivamente (cuadro 5).

Los 20 haplotipos HLA-A,-B,-DRB1 más comunes se muestran en la figura 2. El haplotipo $A^{*} 24 B^{*} 35$ DRB $1 * 04(4,6 \%)$ fue el más frecuente, seguido de $A^{*} 24 B^{*} 61$ DRB1*04 (2,0\%).

\section{Discusión}

Los alelos más comunes para HLA-A fueron $A^{*} 02$, A24, $A^{\star} 03, A^{\star} 23$ con frecuencias similares a las previamente reportadas por nuestro grupo (excepto A23*, que no había sido detectado por serología) y a las frecuencias reportadas en población mestiza colombiana (42). Los resultados están de acuerdo con los distintos reportes de frecuencias alélicas en población hispánica o latinoamericana, en los cuales los alelos de mayor frecuencia fueron: $A^{\star} 02, A^{\star} 24, A^{\star} 03, A^{\star} 30, A^{*} 68$, $A^{*} 01$ (24-27). Sin embargo, la frecuencia de $A^{*} 23$ $(7,2 \%)$ es superior a la observada en el estudio de Cao et al. (1,9\%), en el que se evaluaron 234 sujetos con mezcla génica latinoamericana residentes en Estados Unidos y a la frecuencia observada en el estudio de Mori et al. (2.9\%) en una población de 100.128 latinos $(p<0,0001$ en ambas comparaciones).

El presente estudio permitió determinar un mayor número de alelos en comparación al trabajo realizado anteriormente por nuestro grupo (41), lo cual se puede explicar por las diferencias en las técnicas empleadas para la clasificación HLA. En el pasado se utilizó la técnica serológica de microlinfotoxicidad con la cual $52,7 \%$ de los antígenos HLA-DRB no fueron identificados y se consideraron alelos nulos.

En el presente estudio, en el locus para HLA-B se observó una alta variabilidad genética, 43 alelos, de los cuales, $63,6 \%$ se encontraron con una frecuencia menor de $2 \%$. Los alelos más comunes fueron $B^{\star} 35, B^{\star} 44$ acorde a lo observado en población colombiana (41), uruguaya (29), brasilera (28), hispánica o latinoamericana residente en los Estados Unidos (24-27). Aunque difiere del estudio de Fleischhauer et al. realizado en 59 individuos mestizos de Cali, en el cual los 
alelos de mayor frecuencia fueron $B^{*} 40(13,9 \%)$ y $B^{*} 51(12,5 \%)$, alelos frecuentes en nuestro estudio y en poblaciones hispánica y latinoamericana, pero con frecuencias más bajas.

Con respecto al locus para HLA-DRB, los alelos más comunes (DRB1*04, DRB1*13, DRB1*07, DRB1*15, DRB1*01, DRB1*17, DRB1*11, DRB $\left.{ }^{*} 08\right)$, son los que predominan en población hispánica o latinoamericana de Estados Unidos), aunque la frecuencia de $\mathrm{DRB}^{\star} 08(5,6 \%)$ en nuestro estudio es estadísticamente menor $(p<0,0001)$, a la observada en población hispánica $(8,9 \%)(25)$ y en población latina $(9,8 \%)(27)$.

Las diferencias observadas en algunas frecuencias alélicas entre los distintos estudios son de esperarse, debido a las diferencias en el tamaño de la muestra, técnicas empleadas en la detección de los alelos y a la variación interpoblaciones que puede existir.

La falta de ajuste al principio de Hardy-Weinberg en una población como la de Medellín, en la que se encuentra un déficit de heterocigóticos, puede ser causada por factores tales como: 1) que el locus esté bajo selección, 2) deriva genética, 3) presencia de alelos nulos, 4) endogamia, 5) emparejamiento selectivo, 6) desequilibrio de ligamiento y, finalmente, 7) factores técnicos. De estas siete opciones, el alto desequilibrio de ligamiento entre los loci HLA demostrado en este estudio, la selección natural y, de alguna manera, el emparejamiento selectivo que se ha dado en Antioquia (49), pudieran explicar las desviaciones del equilibrio Hardy-Weinberg encontradas en la distribución de los genotipos para los loci HLA-A y HLA-B, lo cual se sustenta en los índices de fijación de Fisher encontrados para los loci HLAA y B. Sin embargo, cabe mencionar la posibilidad de que la deficiencia de individuos heterocigóticos observados, pueda atribuirse a la falta de detección de alelos por la PCR-SSP, que aunque tiene un mayor grado de resolución que las técnicas serológicas, puede no discriminar algunos alelos dentro de un mismo subgrupo.

Además, se presentó un alto desequilibrio de ligamiento entre los loci, siendo menos pronunciado entre los loci HLA-A y HLA-DRB1. Este hallazgo pudiera ser explicado por el aislamiento genético de la población antioqueña después del período colonial y por el continuo mestizaje entre hombres españoles y mujeres mestizas, como se demostró en un estudio reciente mediante el análisis de marcadores genéticos del cromosoma $Y$ y del ADN mitocondrial (50).

Como era de esperarse, entre los haplotipos más frecuentes, se encuentran los alelos de mayor frecuencia de cada locus, por ejemplo, $A^{\star} 02$ se encontró formando varias combinaciones haplotípicas con alelos del locus para HLA-B y HLA-DRB1.

Al comparar los distintos haplotipos observados en este estudio con otros realizados en poblaciones clasificadas según el grupo étnico, se encontró que estos corresponden a los haplotipos más frecuentes en población hispánica, latinoamericana y caucásica (25-27). Es así como los haplotipos $A^{*} 01 B^{*} 08, A^{*} 24 B^{*} 35, A^{*} 02 B^{*} 44$, $A^{*} 03 B^{\star} 07, A^{\star} 29 B^{\star} 44, A^{*} 03 B 35, A^{*} 02 B^{\star} 51, A^{*} 11$ $B^{\star} 35, A^{*} 02 B^{\star} 35$ y $A^{\star} 26 B^{*} 38$, presentes en población latinoamericana, se encuentran dentro de los 15 más frecuentes en población caucásica, siendo los haplotipos $A^{*} 02 B^{*} 35, A^{*} 24 B^{*} 35$ los más comunes en el grupo de latinos, y $A^{*} 01 B^{*} 08, A^{*} 02$ $B^{*} 44$ los más comunes en caucásicos (25-27).

De igual manera, los haplotipos de mayor frecuencia para HLA-B,-DRB1* se encuentran dentro de los 25 haplotipos más comunes en población hispánica y caucásica, con excepción de B*65 DRB1*13, B*53 DRB1*13; B*35 DRB1*07; siendo las combinaciones haplotípicas de mayor frecuencia en hispanos $B^{*} 35$ DRB $1{ }^{*} 04, B^{*} 44$ DRB1*07, y $B^{*} 8 \mathrm{DRB} 1{ }^{*} 03$ en caucásicos.

Cabe mencionar que aunque los resultados de este estudio están de acuerdo con lo encontrado en otras poblaciones, la distribución de las frecuencias génicas y haplotípicas fue diferente en algunos casos, lo cual evidencia la variación a nivel interpoblación que puede existir. Por lo tanto, la realización de este estudio nos permitió conocer nuestras frecuencias génicas, información de gran utilidad para estudios antropogenéticos, de asociaciones entre HLA y enfermedades, en el cálculo de la probabilidad de encontrar un donante compatible para determinado receptor. Por otra parte, se corrobora la mezcla racial existente en 
nuestra población, típica de población latinoamericana pero con un alto grado de influencia caucásica (51).

\section{Agradecimientos}

Los autores agradecen a Gabriel Bedoya por la lectura crítica de este manuscrito y a todos los miembros de los grupos de trasplantes de la ciudad de Medellín.

\section{Conflictos de intereses}

Los autores declaran no tener ningún conflicto de interés en el presente estudio.

\section{Financiación}

Recursos propios del grupo.

\section{Referencias}

1. Marsh S, WHO Nomenclature Committee for Factors of the HLA System. Nomenclature for factors of the HLA system, update September 2006. Hum Immunol. 2006;67:1022-4.

2. Bontrop RE. Comparative genetics of MHC polymorphisms in different primate species: Duplications and deletions. Hum Immunol. 2006;67:388-97.

3. Alper CA, Larsen CE, Dubey DP, Awdeh ZL, Fici DA, Yunis EJ. The haplotype structure of the human major histocompatibility complex. Hum Immunol. 2006;67:73-84.

4. Claas F, Roelen DL, Mulder A, Doxiadis II, Oudshoorn M, Heemskerk M. Differential immunogenicity of HLA Class I alloantigens for the humoral versus the cellular immune response: "Towards tailor-made HLA mismatching". Hum Immunol. 2006;67:424-9.

5. Medema JP, Borst J. T cell signaling: A decision of life and death. Hum Immunol. 1999;60:403-11.

6. Price P, Keane NM, Stone SF, Cheong K, French MA. MHC haplotypes affect the expression of opportunistic infections in HIV patients. Hum Immunol. 2001;62:157-64.

7. Caputo M, Cerrone GE, López AP, González C, Mazza C, Cedola N, et al. Genotipificación del gen HLA DQB1 en diabetes autoinmune del adulto (LADA)/ HLA DQB1 genotyping in latent autoimmune diabetes of adults (LADA). Medicina (B. Aires) 2005;65:235-40.

8. Morera LM, Marsan V, Villaescusa R, Guerrero AM, Figueredo M, Roque M. HLA y leucemias. Estudio de 144 casos. Rev Cuba Hematol Inmunol Hemoter. 1997;13:27-37.

9. Montoya F, Bedoya $\mathrm{Cl}$, Restrepo MC, Villegas A, Posada SC, García H, et al. Determinación de marcadores genéticos en pacientes con diabetes tipo I y población sana. Acta Méd Colomb. 1996;21:10-6.

10. Garavito G, Iglesias A, Egea E, Jaraquemada D, Martínez P, Egea EE. Una aproximación al significado biológico del polimorfismo del complejo mayor de histocompatibilidad. El modelo de la asociación HLA y ARJ. SaludUninorte. 2002;16:53-72.

11. Domínguez MC, Lorenzo N, Barbera A, Hernández MV, Torres AM, Nazabal M, et al. Caracterización de moléculas HLA tipo II y evaluación de citocinas en pacientes cubanos con artritis reumatoide. Rev Cub Reumatol. 2006;8:9-10.

12. Rioboo M, Bascones A, Riobó R. El HLA y su implicación en odontología. Av Odontoestomatol. 2005;21:95-107.

13. Garavito G, Malagón C, Ramírez L, De la Cruz OF, Uribe O, Navarro E, et al. Polimorfismo de los alelos de los antígenos de leucocitos humanos HLA-DRB1 y su asociación con la artritis reumatoidea juvenil en una muestra de niños mestizos colombianos. Biomédica. 2003;23:254-62.

14. Subklewe M, Marquis R, Choquet S, Leblond V, Garnier JL, Hetzer R, et al. Association of human leukocyte antigen haplotypes with posttransplant lymphoproliferative disease after solid organ transplantation. Transplantation. 2006;82:1093-100.

15. Cortes LM, Baltazar LM, Lopez M, Olivares N, Ramos C, Salazar M, et al. HLA class II haplotypes in Mexican systemic lupus erythematosus patients. Hum Immunol. 2004;65:1469-76.

16. Murata M, Emi N, Izumisawa $\mathbf{Y}$, Inaki A, Saitoh $\mathbf{M}$, Naoe T. Identification and frequency of a new HLA-A allele, $A^{*} 030104$. Tissue Antigens. 2005;65:391-2.

17. Witter K, Albert T, Zahn R, Kauke T. A novel HLADQB ${ }^{*} 06$ allele, DQB $1{ }^{*} 0628$, found through routine sequence-based HLA typing and confirmation of DQB1*060302. Tissue Antigens. 2006;69:102-3.

18. Danzer M, Polin H, Schröder S, Mytilineos J, Gabriel C. HLA-Cw*0740, a new allele mistyped by generic sequencing and identified by allelic separation. Tissue Antigens. 2006;69:100-2.

19. Collins MM, Tang T, Slack R, Sintasath D, Hartzman $\mathbf{R J}, \mathbf{N g ~ J}$, et al. The relative frequencies of HLADRB $1{ }^{*} 01$ alleles in the major US populations. Tissue Antigens. 2000;55:48-52.

20. Balas A, García F, Vicario JL. Sequencing of a novel HLA-A allele, $A^{*} 6836$, showing a Bw4 epitope. Tissue Antigens. 2006;69:99-100.

21. Witter K, Wittmann G, Lehmeier AM, Albert T, Kauke T. A novel HLA-DRB $1{ }^{*} 11$ allele, DRB $1 * 1155$, was found in a patient through routine DRB1 typing using sequence-specific primers (SSP) and confirmed by allele-specific sequence-based typing. Tissue Antigens. 2006;68:531-3. 
22. Voorter C, Gervais T, Palusci F, Latinne D, van den Berg-Loonen EM. A novel HLA-A*11 allele, $A^{*} 1118$, identified by sequence-based typing. Tissue Antigens. 2006;68:524-6.

23. Tu B, Mack SJ, Lazaro A, Lancaster A, Thomson G, Cao K, et al. HLA-A, -B, -C, -DRB1 allele and haplotype frequencies in an African American population. Tissue Antigens. 2006;69:73-85.

24. Cao K, Hollenbach JA, Shi XJ, Shi WX, Chopek M, Fernandez-Viña MA. HLA-A, -B and -Cw allele frequencies in a hispanic population from the USA. Hum Immunol. 2004;65:1206-8.

25. Mori M, Beatty PG, Graves M, Boucher KM, Milford EL. HLA gene and haplotype frequencies in the North American population: The National Marrow Donor Program Donor Registry 1. Transplantation. 1997;64: 1017-27.

26. Cao k, Hollenbach J, Shi X, Shi W, Chopek M, Fernández-Viña MA. Analysis of the frequencies of HLA-A, B, and C Alleles and haplotypes in the five major ethnic groups of the United States reveals high levels of diversity in these loci and contrasting distribution patterns in these populations. Hum Immunol. 2001; 62:1009-30

27. Zachary AA, Steinberg AG, Bias WB, Leffell MS. The frequencies of HLA alleles and haplotypes and their distribution among donors and renal patients in the UNOS registry. Transplantation. 1996;62:272-83

28. Monte S, Moita J, Rampim G, Shulzhenko N, Morgun A, Gerbase-deLima M. Polimorfismo do sistema HLA em uma amostra de mestiços da população de Teresina, Piauí. Rev Assoc Méd Brás. 2004;50:422-6.

29. Bengochea M, Álvarez I, Hidalgo PC,Cabrera A, Senatore 0, Toledo R, et al. HLA-A, -B, -DR en receptores de trasplante de médula ósea en Uruguay. Rev Med Uruguay. 2003;19:149-58.

30. Arango A, Camacho GP. La antropología genética de la población Colombiana. Exhumar. 2005;2:66-78.

31. Middleton D, Williams F, Meenagh A, Daar AS, Gorodezki C, Hammond $\mathbf{M}$, et al. Analysis of the distribution of HLA-A alleles in populations from five continents. Hum Immunol. 2000;61:1048-52.

32. Williams F, Meenagh A, Darke C, Acosta A, Daar AS, Gorodezky C, et al. Analysis of the distribution of HLA-B alleles in populations from five continents. Hum Immunol. 2001;62:645-50.

33. Pédron B, Yakouben K, Guérin V, Borsali E, Auvrignon A, Landman $\mathrm{J}$, et al. HLA alleles and haplotypes in French North African Immigrants. Hum Immunol. 2006;67:540-50.

34. Cao K, Hollenbach J, Shi X, Shi W, Chopek M, Fernandez-Viña MA. Analysis of the frequencies of HLA-A, B and C alleles and haplotypes in the five major ethnic groups of the United States reveals high levels of diversity in these loci and contrasting distribution patterns in these populations. Hum Immunol. 2001;62:1009-30.

35. Little AM, Scott I, Pesoa S, Marsh SG, Arguello R, Cox ST, et al. Class I diversity in Kolla Amerindians. Hum Immunol. 2001;62:170-9.

36. Goodman RS, Taylor CJ, O'Rourke CM, Lynch A, Bradley JA, Key T. Utility of HLAMatchmaker and single-antigen HLA-antibody detection beads for identification of acceptable mismatches in highly sensitized patients awaiting kidney transplantation. Transplantation. 2006;81:1331-6.

37. Duquesnoy RJ, Askar M. HLAMatchmaker: A molecularly based algorithm for histocompatibility determination. V. Eplet Matching for HLA-DR, HLA-DQ, and HLA-DP. Hum Immunol. 2007;68:12-25.

38. Duquesnoy RJ, Claas F. Is the application of HLAMatchmaker relevant in kidney transplantation? Transplantation. 2005;79:250-1.

39. Duquesnoy RJ. A structurally based approach to determine HLA compatibility at the humoral immune level. Hum Immunol. 2006;67:847-62.

40. Gil P, Poutou R, Ossa H. Frecuencias génicas del sistema HLA clase I y II en una población de la ciudad de Bogotá D.C. [Consultado: 5 de septiembre de 2006]. Disponible en: http://www.monografias.com/trabajos12/ arthla/arthla.shtml

41. París SC, García LF. El complejo mayor de histocompatibilidad humano. Sistema HLA. latreia. 1989;2:137-55

42. Fleischhauer K, Zino E. HLA-A, -B and -Cw allele frequencies in two populations from Colombia. Human Immunol. 2004;65:906-8.

43. Trachtenberg EA, Keyeux G, Bernal JE, Rodas MC, Erlich HA. Results of Expedición Humana. I. Analysis of HLA class II (DRB1-DQA1-DQB1-DPB1) alleles and DR-DQ haplotypes in nine Amerindian populations from Colombia. Tissue Antigens. 1996;48:174-81.

44. Trachtenberg EA, Keyeux G, Bernal JE, Noble JA, Erlich HA. Results of Expedición Humana. II. Analysis of HLA class II alleles in three African American populations from Colombia using the PCR/SSOP: Identification of a novel DQB $1{ }^{*} 02\left({ }^{*} 0203\right)$ allele. Tissue Antigens. 1996; 48:192-8.

45. Hahn AB, Land GA, Strothman RM. ASHI Laboratory Manual. Fourth ed. Lenexa: American Society for Histocompatibility and Inmunogenetics. DigiScript Inc; 2000.p.713-8.

46. Raymond M, Rousset F. GENEPOP (version 1.2): population genetics software for exact tests and ecumenicism. J Hered. 1995;86:248-9.

47. Excoffier L, Laval G, Schneider S. Arlequin ver. 3.0: An integrated software package for population genetics data analysis. Evol Bioinform Online. 2005;1:47-50. 
48. Weir BS. Genetic Data Análisis II. 2da. Sunderland (MA): Sinauer Associates; 1996.

49. Centro de Investigación y Educación Popular. Región Noroccidental. En: Colombia, país de regiones. Tomo I. Santafe de Bogotá: CINEP; 1998. [Consultado: 6 de diciembre de 2006]. Disponible en: http://lablaa.org/ blaavirtual/geografia/region1/present.htm.

50. Carvajal-Carmona LG, Soto ID, Pineda N, OrtizBarrientos D, Duque C, Ospina-Duque J, et al.
Strong Amerind/White sex bias and a possible sephardic contribution among the founders of a population in northwest Colombia. Am J Hum Genet. 2000;67:1287-95.

51. Bedoya G, Montoya P, García J, Soto I, Bourgeois $\mathrm{S}$, Carvajal L, et al. Admixture dynamics in Hispanics: A shift in the nuclear genetic ancestry of a South American population isolate. Proc Natl Acad Sci USA. 2006;103:7234-9. 